\title{
Effect of Temperature on Some Rheological Properties of Polyvinyl Pyrrolidone (PVP)
}

\author{
Suzanne R. Aboud ${ }^{1}$, Abdul Kareem1 J. Al-Bermany ${ }^{2}$ \\ and Nassar Megahed ${ }^{3}$ \\ ${ }^{1)}$ Physics Department, Faculty of Science, Al- Gabal Al- Gharbi \\ University, Libya. \\ 2) Physics Department, Faculty of Science, Babylon University, Iraq. \\ 3) Physics Department, Faculty of Science, Sohag University, Egypt.
}

Some of the rheological properties of polyvinyl pyrrolidone (PVP) as a function of temperature had not been studied. The relative, specific, reduced, intrinsic viscosities, effective molecular radius and molecular weight were determined in this work. Different dilute concentrations dissolved in distilled water using Ostwald Viscometer have been used. Results show that shear, relative, specific, reduced viscosities increase with increasing the polymer concentrations and also show that these types of viscosities decrease with increasing the temperature. Results also show that intrinsic viscosity and molecular weight values that have been experimentally obtained have good agreement with the theoretical values which were obtained by the use of Pilipoff and Arrhenus equations. Hence, it is concluded that the method has a high precision for estimating intrinsic viscosity and molecular weight.

\section{Introduction:}

Polyvinyl pyrrolidone is made from the monomer n-vinyl pyrolidone. The monomer is carcinogenic and is extremely toxic to aquatic life. However its polymer PVP in its pure form is also safe that not only is it edible by humans, but also is used as a blood plasma expander for trauma victims. PVP is soluble in water and other polar solvent. In water it has the useful property of Newtonian viscosity. When dry it is a light flaky powder, which readily absorbs up to $18 \%$ of its weight in atmospheric water. In solution, it has excellent wetting properties and readily forms films. This makes it good as a coating or an additive to coatings. The uses to which PVP has been put are very wide. It is used as a binder in many pharmaceutical tablets, being completely inert to humans. PVP binds to polar molecules exceptionally well, owing to its polarity. This has led to its application in coatings for photo-quality ink-jet papers and transparencies, as well as in inks for ink-jet printers also used in shampoos, toothpastes, adhesives [1]. 
The viscosity of a liquid is associated with the interaction between molecules and manifests itself as a resistance to flow [2]. The theoretical background for the use of viscosity measurements to study macromolecules started with Einstain's relationship of equation relating the viscosity of a dilute suspension of small rigid spheres to the volume fraction $\phi$ occupied by the spherical particles as follows: $\eta_{s p}=2.5 \phi$ where $\eta_{s p}$ is the specific viscosity.

It is known that most synthetic polymer molecules consist of chains of monomer units and are threadlike and flexible in solution. The temperature is very effective on these chains and gives its molecules high values of flexibility by reducing strength of molecules bounds.

As the intrinsic viscosity depends on the molecular weight, so, MarkHouwink relation was used in this study to measure the molecular of this polymer [2- 4] as follows:

$$
(\eta)=K M^{a}
$$

where $K$ and $a$ are two parameters that depend on the polymer and solvent.

\section{Experimental:}

The solutions for different concentrations of (PVP) were prepared by adding known weights of the polymer to a fixed volume of distilled water and heating the mixture under flux whilst agitating with magnetic stirrer.

The viscosity of the different dilute concentrations was measured by using Ostwald Viscometer. Double walled chambers of suitable size were prepared for measurement and water from a thermostatic bath Lay-bold (Germany) was constantly circulated through these chambers for different temperature ranges of 25,35 and $45^{\circ} \mathrm{C}$.

The flow time $t_{o}$ of the distilled water and of the polymer solution $t$ for different concentrations was recorded. The density $\rho$ of these solutions was measured by using a well known density bottle method (bicknometer). 


\section{Theoretical:}

Shear viscosity $\eta_{s}$ of each concentration was determined by the [2, 3],

$$
\frac{\eta_{s}}{\eta_{o}}=\frac{t_{s} \rho_{s}}{t_{o} \rho_{o}}
$$

where $\rho_{s}$ is the solution density, experimentally obtained. The distilled water density $\rho_{o}$ and viscosity $\eta_{o}$ were taken from tables at different temperature [4]. Relative viscosity $\eta_{\text {rel }}$ has been calculated using the following equation [3-5],

$$
\eta_{\text {rel }}=\frac{\eta_{s}}{\eta_{o}}
$$

The specific viscosity $\eta_{s p}$ was calculated from:

$$
\eta_{s p} \frac{\eta_{s}}{\eta_{o}}-1
$$

The reduced viscosity $\eta_{\text {red }}$ has been calculated using the following equation:

$$
\eta_{\text {red }}=\frac{\eta_{s p}}{C}
$$

where $C$ is the polymer concentration. The effective molecular radius $r$ was calculated using Eqn. (7) $[4,5]$.

$$
\eta_{s p}=2.5\left(\frac{4}{3} \pi N_{A}\right) \cdot r^{3} C
$$

where $N_{A}$ is Avogadro's number, C is molar concentration in (mole/ml) unite and equal to $C=\frac{W}{M V}$, where $W$ is the polymer weight, $M$ is the molecular weight and $V$ is the solution volume.

$$
\eta_{\text {rel }}-1=6.3 \times 10^{24} r^{3} \mathrm{C}
$$

and

$$
r=\left(\frac{\text { slope }}{6.3 \times 10^{24}}\right)^{1 / 3}
$$

which can be calculated from Fig. (3) for different temperatures. The intrinsic viscosity $\eta$ was theoretically calculated by using Arrhenius equation [6], $\ln \eta_{\text {rel }}=\eta C$, and also calculated by using the Pilipoff [6],

$$
\eta_{\text {rel }}=\left(1+\eta \frac{C}{8}\right)^{8}
$$




\section{Results and Discussion:}

The results of shear viscosity against concentration are shown in Fig. (1). This figure shows that the viscosity is increasing with the increasing of the concentration. This behavior of viscosity is like that given by Adam and Gatta [4] and Dooley [7] for other polymers. This is attributed to the fact that increasing the polymer concentration supports complex formation between polymer molecules and solvent molecules and partially due to attachment by hydrogen bounding of water (solvent) to the Oxygen sites. As polymer resolved in solute, its macromolecules have randomly coiled. As the concentration increases the polymer molecules becomes large, so it impedes its luminaries against the flow leading to an increase in the flow time $[5,8]$. The solubility mechanism can lead to salvation the effect of the layers and increase the size of the molecules with subsequent increase in the viscosity [5].

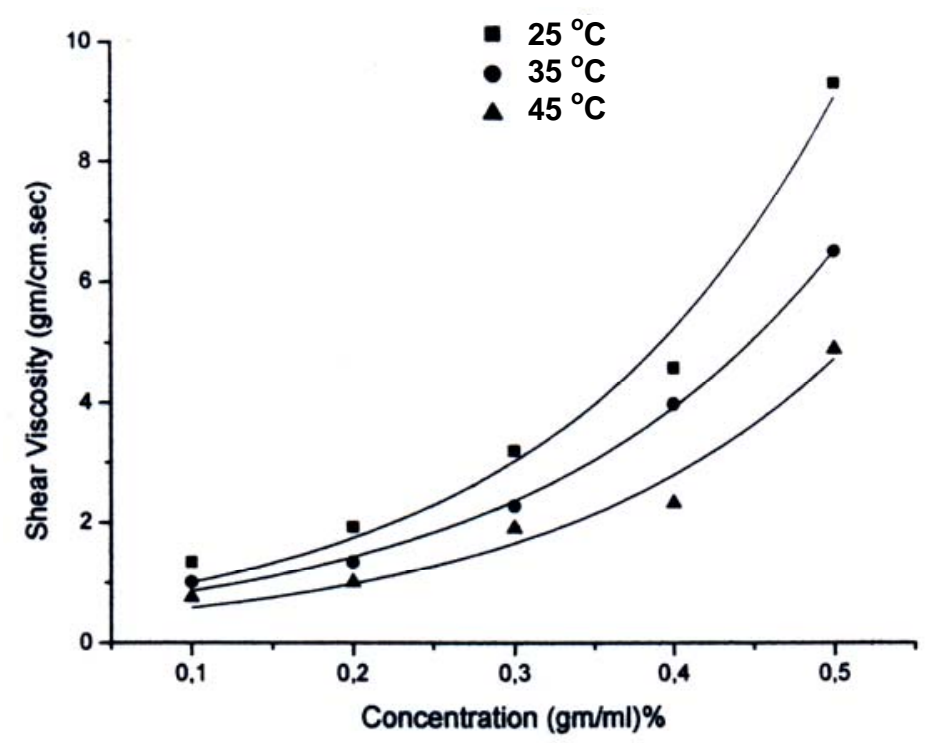

Fig. (1): Shear viscosity vs. concentration with different temperatures.

As temperature $T$ increases the viscosity decreases as shown in the same figure because the temperature would give bond molecules energy which make them tightly bond so have much flexibility to flow through capillary tube. This result agrees with theoretical equation of exponential law given by [9]: $\eta=\eta_{\text {ref }} \exp (-b \Delta t)$. The value of the temperature sensitivity $b$ was calculated by plotting the relative viscosity against temperature. The value was found to be equal to $0.02{ }^{\circ} \mathrm{C}^{-1}$. Other polymer range from about 0.01 to $0.1{ }^{\circ} \mathrm{C}^{-1}$ was reported by Macosko [9]. 
Figure (2) shows that density increase linearly with the concentration. These behaviors are similar to that given by $[5,8]$ for other polymers. This is attributed to the fact that as polymer weight uniformly increases the density also uniformly increases because the density is the ratio between increasing polymer weight to fixed volume of solution [5]. This figure shows that when temperature increases the density decreases. This is because the temperature increases the molecules kinetic energy leading to change their conformation and configuration [10]. This reduces the solute polymer to affixed volume.

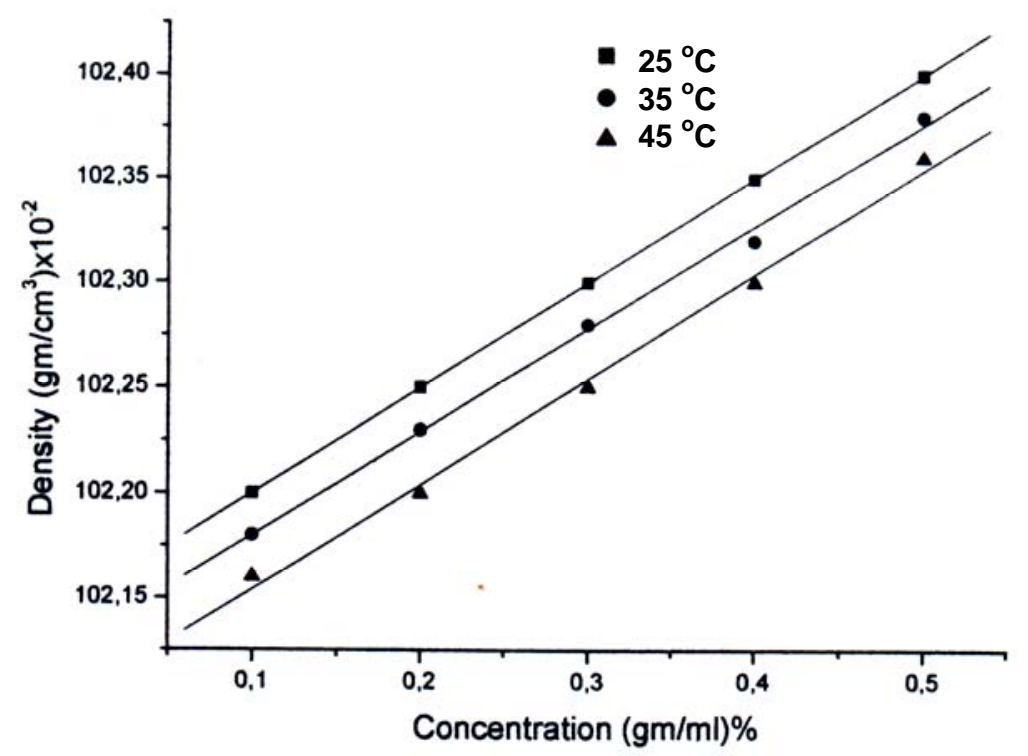

Fig. (2): Density vs. concentration with different temperatures.

Other rheological properties such as relative and specific viscosities shown in Figs. ( $3 \& 4$ ) were derived from shear viscosity and they have the same behavior of increasing shear viscosity with increase in concentration [11]. Fig. (5) shows the reduced viscosity against concentration, when the extrapolation of this slope as concentration goes to zero gives intrinsic viscosity $\eta$ value as given in Table (1). The theoretical values of the intrinsic viscosity were calculated by using Arrhenius and Philipoff equations. The comparison between the experimental and the theoretical values of the intrinsic viscosity obtained by these two equations are shown in Table (1). 


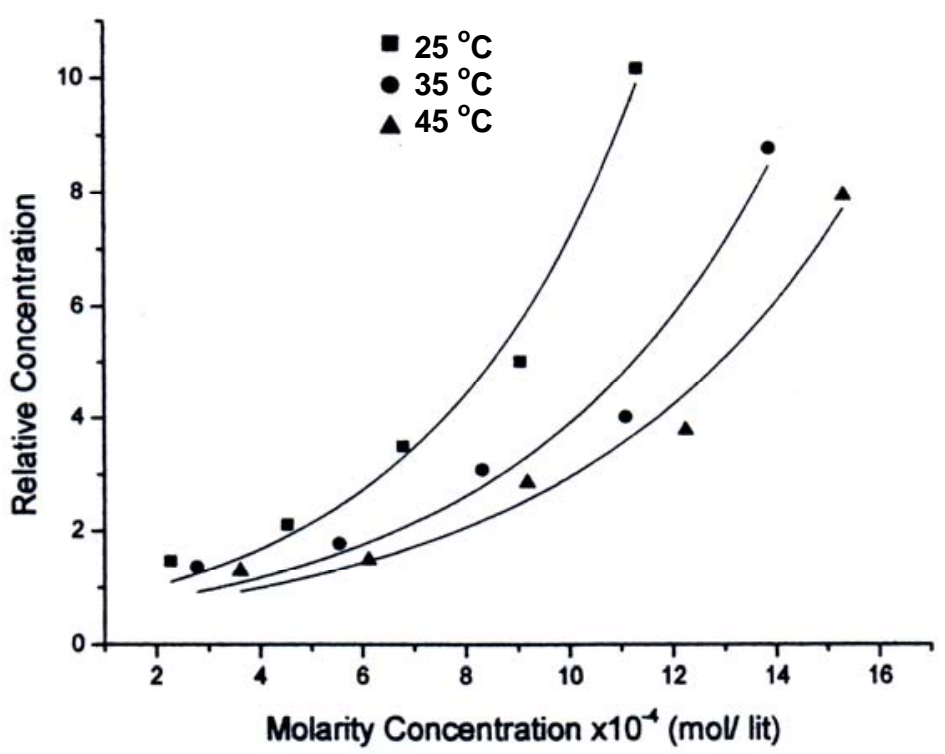

Fig. (3): Relative viscosity vs. concentration with different temperatures.

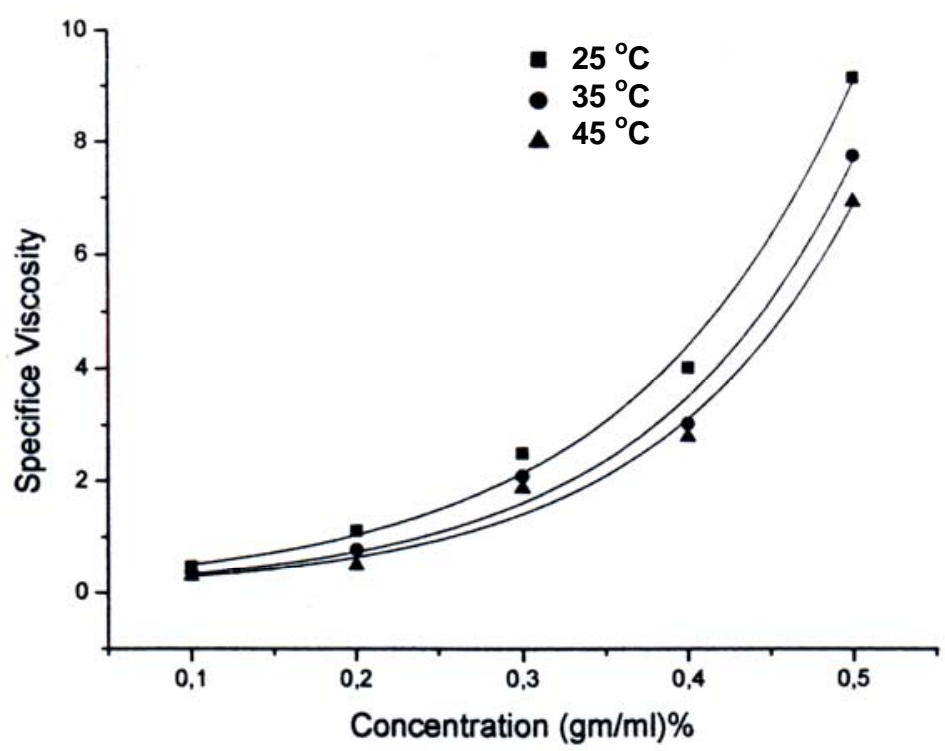

Fig. (4): Specific viscosity vs. concentration with different temperatures. 
Fig. (5)

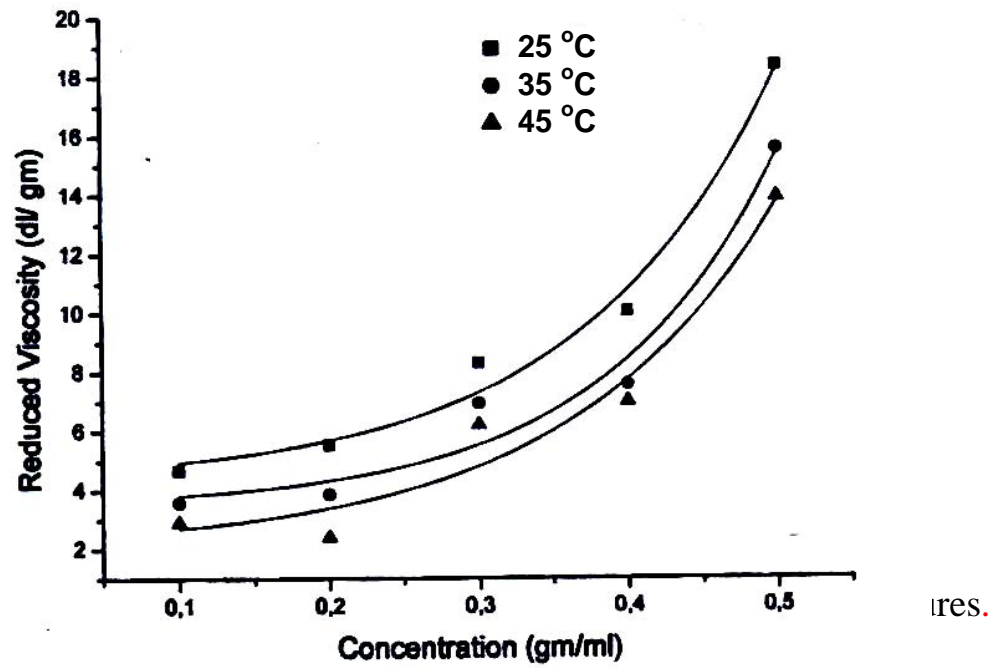

Table (1): Comparison between theoretical and experimental values of intrinsic viscosity, molecular weight and effective molecular radius with different temperature.

\begin{tabular}{|c|c|c|c|c|c|c|c|c|}
\hline \multirow{3}{*}{$\begin{array}{l}\text { Temp || } \\
\left({ }^{\circ} \mathrm{C}\right)\end{array}$} & \multicolumn{3}{|c|}{$\begin{array}{l}\text { Intrinsic Viscosity } \\
\quad(\mathrm{ml} / \mathrm{gm})\end{array}$} & \multicolumn{3}{|c|}{$\begin{array}{l}\text { Viscosity Average } \\
\text { Molecular Weight } \\
(\mathrm{gm} / \mathrm{mol})\end{array}$} & \multicolumn{2}{|c|}{$\begin{array}{c}\text { Effective Molecular } \\
\text { Radius } \\
(\mathrm{cm}) \\
\end{array}$} \\
\hline & \multicolumn{2}{|c|}{ Theoretical } & \multirow{2}{*}{ Expt. } & \multicolumn{2}{|c|}{ Theoretical } & \multirow{2}{*}{ Expt. } & \multirow{2}{*}{$\begin{array}{l}\text { Low } \\
\text { Concentration } \\
\left(\mathrm{x} 10^{-8}\right)\end{array}$} & \multirow{2}{*}{$\begin{array}{l}\text { High } \\
\text { Concentration } \\
\left(\times 10^{-7}\right)\end{array}$} \\
\hline & Arrh. & Philip. & & Arrh. & Philip. & & & \\
\hline 25 & 4.076 & 4.30 & 4.2 & 380305 & 406607 & 394822 & 10.060 & 1.238 \\
\hline 35 & 3.496 & 3.60 & 3.5 & 313909 & 325625 & 314358 & 9.128 & 1.300 \\
\hline 45 & 3.090 & 3.29 & 3.1 & 269022 & 290961 & 270110 & 8.982 & 1.070 \\
\hline
\end{tabular}

The effective molecular radius of this polymer was calculated by using Eqn. (7) and Fig. (3). Their values are given in Table (1). The average molecular weight, $M_{v}$, was obtained by using Mark-Houwink equation. Our experimentally obtained values are in good agreement with the theoretical values of Arrehenius and Philipoff equations with some acceptable experimental errors. 


\section{Conclusions:}

Increasing polymer weight uniformly increases the density and the viscosity grade. The density decreases when the temperature increases, this is because the temperature increases the molecules kinetic energy leading to change its conformation and configuration. Increase in the temperature reduces the molecular weight and its viscosity grade as a result of tightly bond molecules. We can obtain different grades of molecular weight by changing the temperature. This could be used in different applications.

\section{References}

1. www.reference.Com/ browse/ Wiki/poly_vinyl prrolidone.

2. J. Brandup, E. H Immergut and W. McDowell, eds," Polymer Hand Book" second edition, Wily-Intescience, New York, (1975).

3. P, J, Flory, "Principle of Polymer Chemistry" Cornell University Press, Ithaca, New York, (1953).

4. G. A. Adam, H. K. Al. Gatta," Chemistry and Technology of Polymer" Al. Basra University Press, Iraq, (1983).

5. Al. Bermany, A. K. J., Ph. D. thesis, Al. Mustansirya University, (1995).

6. M. Norbert and L. Leons, "Cellulose and Cellulose Derivative", V, part. V, Jhom W. And sons, Inc., (1971).

7. J. Dooley, Ph. D. thesis, University of Eindhoven, Netherlands (2002).

8. Al. Ebaidy, Z. C., M. Sc. thesis, Baghdad University (1995).

9. C. W. Macosko, "Rheology: Principle, Measurement and Applications", VCH publishers, New York (1994).

10. H. Gharibi et al. "Colloids Surfaces A: Physicochem". Eng. Aspects (1998).

11. F. N. Cogswell, "Polymer Melt Rheology", Woodhead Puplishing, Cambridge, England (1996). 